\title{
Finite Element Analysis of Bulge Forming of Laser Welding Dimple Jacket
}

\author{
Peisi ZHONG ${ }^{1 *}$, Shile MA ${ }^{1}$, Yunkai YANG ${ }^{1}$, Haiyan WANG ${ }^{2}$, Runqiang LIU $^{2}$, \\ Yuyan SUN ${ }^{1}$ \\ ${ }^{1}$ Advanced Manufacturing Technology Center, Shandong University of Science and Technology, Qingdao 266590, China \\ ${ }^{2}$ College of Mechanical and Electronic Engineering, Qingdao Huanghai University, Qingdao 266427, China \\ cross'ref http://dx.doi.org/10.5755/j01.ms.21.4.9704
}

Received 28 January 2015; accepted 16 November 2015

\begin{abstract}
The stress-strain states of the model of laser welded dimple jacket is analyzed using ANSYS/LS-DYNA in order to determine the relation between bulging height and pressure and to achieve the controllability of pressure distension of the jacket. It is shown that in the same conditions, the bulging height increases with the increasing of the bulging pressure and the space of honeycomb. And it will decrease when the thickness of jacket plate changing larger. A table showing the relation between bulging height and pressure is obtained. An experiment using a test panel is conducted to certify the reliability of finite element analysis. It turns out that the data of finite element analysis is coincident with experimental data, which support finite element method based ANSYS/LS-DYNA can be an efficient way to research the laser welded dimple jacket. The relation table is useful as guidance for the fabrication process.

Keywords: laser welded dimple jacket, bulging forming, finite element, ANSYS/LS-DYNA, bulging pressure.
\end{abstract}

\section{INTRODUCTION}

Laser welded dimple jacket is a large steel heat exchanger with a wide application prospect. As a kind of cooling heat exchange device like Wine fermenter and yeast culture tank, it is mainly used for chemical industry, light industry, and some other industries. In process industry, we often need to add a jacket outside the process equipment to speed up the transmission of heat and the overall jacket is the type we once used most. Overall jacket has the advantages of simple structure, convenient repair and low cost. While because of the low heat transfer coefficient, overall jacket cannot meet the requirements anymore when the dimensions of process equipment become larger and larger. Laser welded dimple jacket is an improved version of overall jacket, which has higher heat transfer coefficient and advanced structure [1].

The structure of laser welded dimple jacket is shown in Fig. 1. Two plates with different thickness are included in the structure. The shell is much thicker than the jacket, so it is the Main body of pressure vessel. The jacket is attached to the shell to cool or heat the shell. In the production process, we need to put the jacket and shell jointed together and then spot welding them together along the points that arranged in equilateral triangle layout. Roll the integral plate into a barrel and make the jacket bulging by pressing water into the space between the shell and the jacket. In the end, we inject the heat transfer medium into the jacket and make the medium circulatory. The fluid in the honeycomb will flow in high speed and get the disturbance of welding circle. So this kind of jacket has the advantage of transferring heat in high coefficient [2].

It is a key step to use bulging forming in laser welded dimple production. During the jacket bulging process, the bulging pressure corresponding to the desired height is

\footnotetext{
* Corresponding author: Tel:+370-37-13789870651.

E-mail address: pszhong@163.com (P. S. Zhong)
}

necessary and the relationship between the bulging height and pressure should be known to avoid the rupture of the jacket caused by high speed bulging and excessive pressure [3]. LS-DYNA program, as the world's most famous generic explicit dynamic analysis program, can simulate any complex structure calculation problems. It is particularly suitable for solving problems of high-speed collision, penetration and explosion shock which are varieties of two-dimensional and three-dimensional nonlinear structure. The reliability of calculation has been demonstrated in numerous tests. It has been widely recognized to be not only the best generic nonlinear finite element analysis program in engineering applications, but also a combination program of military and civilian [4].

\section{EXPERIMENTAL DETAILS}

As a heat-transfer equipment, the jacket usually uses alcohol, water vapour, liquid ammonia as heat transfer medium under different heat requirements [5]. According to engineering experience, different heat transfer medium has different thickness requirements for the jacket plate which is shown in Table 1.

Table 1. Different thickness requirement of different heat transfer medium

\begin{tabular}{|c|c|c|c|}
\hline $\begin{array}{c}\text { Heat } \\
\text { transfer } \\
\text { medium }\end{array}$ & $\begin{array}{c}\text { Working } \\
\text { pressure, MPa }\end{array}$ & $\begin{array}{c}\text { Design } \\
\text { pressure, MPa }\end{array}$ & Thickness, mm \\
\hline alcohol & 0.4 & 0.6 & 1.0 \\
\hline $\begin{array}{c}\text { water } \\
\text { vapor }\end{array}$ & 0.4 & 0.6 & 1.2 \\
\hline $\begin{array}{c}\text { liquid } \\
\text { ammonia }\end{array}$ & 0.4 & 1.3 & 1.2 \\
\hline
\end{tabular}

In addition to the requirement of thickness, some other parameters should be concerned, such as the arrangement style of the dimple elements, the cellular spacing, diameter of the welding ring, the width and height of the dimple. In 
order to obtain the general process of the dimple jacket's pressure bulging, a regular triangle was used as the shape model of the dimple unit. A series of parameter combinations were selected for analysis. The model parameter combinations are shown in Table 2. For example, the model parameter 88_20_1.0 means the edge length is $88 \mathrm{~mm}$, the diameter of the welding circle is $20 \mathrm{~mm}$, and the thickness of the jacket is $1.0 \mathrm{~mm}$. To facilitate the modelling and analysis, the width parameters of the weld is ignored on the premise of avoiding affecting the accuracy.
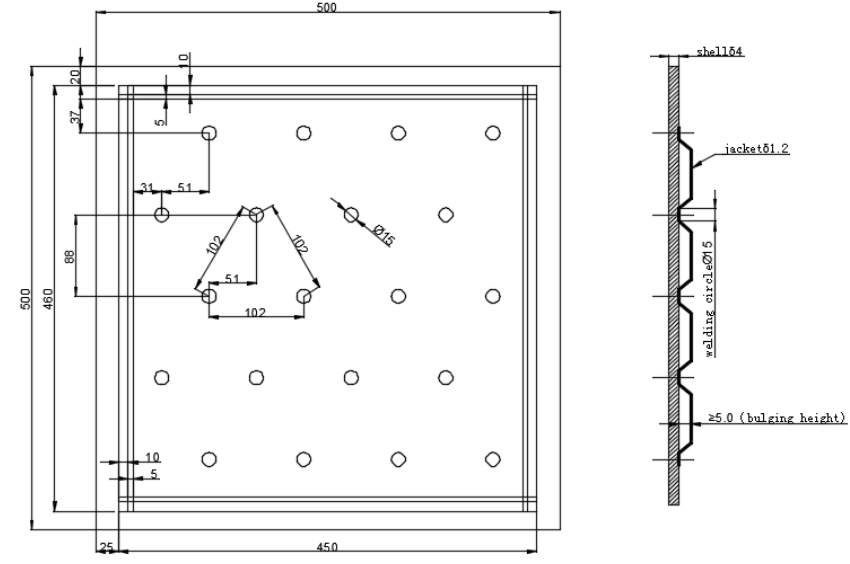

Fig. 1. The structure of the jacket

Table 2. A series combination of parameters

\begin{tabular}{|c|c|}
\hline Heat transfer medium & Model parameters \\
\hline \multirow{2}{*}{ Alcohol } & $88 \_20 \_1.0$ \\
\cline { 2 - 2 } & $100 \_20 \_1.0$ \\
\hline \multirow{2}{*}{ Water vapor } & $88 \_20 \_1.2$ \\
\cline { 2 - 2 } Liquid ammonia & $100 \_20 \_1.2$ \\
\cline { 2 - 2 } & $60 \_15 \_1.2$ \\
\hline
\end{tabular}

Take 88_20_1.0 as an example, use the bottom-up modelling approach to create a solid model of the jacket unit in LS-DYNA. As we can see in the structure of the jacket, the jacket is welded in the shell using energydensity laser beam. So the boundary of the welding circle was considered as be fixed. Meanwhile, considering the symmetry of the structure and for the aim of fast calculation, we just need to select a smallest unit of structure as the finite element model. The horizontal displacement of the equilateral triangle model is zero. So zero-displacement constraints were applied in the edge of the model [6]. In ANSYS/LS-DYNA, many of the standard commands are invalid. Under the circumstance of ANSYS, node coordinate system can be converted into local coordinate system defined by EDLCS by using the command 'csys'. While in LS-DYNA, when the constraints are not coincident to Cartesian coordinate system, it is not allowed to convert it's node coordinate system into user-defined coordinate system to impose these constraints. Local coordinate system defined by EDLCS is implicit coordinate system, so it can not be used in explicit coordinate system. ANSYS/LS-DYNA provides different commands named EDNROT and EDLOAD to achieve this function. In addition, the constraints and loads defined by EDNROT and EDLOAD also can not be revealed using ANSYS command. ANSYS/LS-DYNA provides the commands of EDLCS and EDNROT to define coordinate system and constraint condition. For the same nodes, it is not allowed to define the constraint condition in two different coordinations at the same time. So it must be avoided to repetitively impose constraints for nodes. The definition of constraints is shown in Fig. 2.

Solid model is established in the front stage in LSDYNA, including the definition of unit characteristics, real constants, material properties and the mesh.

Because the bulging forming will cause large deformation, and plastic deformation is producing in bulging process, so shell-163 unit type is selected. Shell163 is an explicit shell unit used in kinetic analysis only. With bending and membrane characteristics, it can add the shear load and normal load. The Belytschko-Wong algorithm, which is suitable for metal forming analysis, was chosen as the unit algorithm. Assigning a value to every real constant of Shell-163 is necessary. The shear factor SHRF is $5 / 6$, the numerical integral point of unit thickness NIP is 5. The thickness of jacket model is uniform, so only the value of T1 need to be specified to the thickness of the jacket [7].

Bilinear Kinematic Hardening was chosen for the material model, which can simulate the constitutive relation of elastic-plastic material by two straight line segments. The relations between material stress and strain are proportional before the material is yield. While after that, the relations change according to tangent modulus. The model has two slopes: the elastic slope and plastic slope. The unload process and spring back can not be simulated if rigid-plastic material model is used in metal forming simulation. So the residual stress can not be predicted too. While there are both elastic deformation and plastic deformation in elastic-plastic deformation forming process and in this case elastic-plastic material model is more suitable for the real metal forming situation. The material model parameters including density, elastic modulus, Poisson's ratio, the yield strength and the tangent modulus are necessary. The material of the laser welded dimple jacket is SUS304 stainless steel (correspond to domestic 0Cr18Ni9), having a density of $7930 \mathrm{~kg} / \mathrm{m}^{3}$, modulus of elasticity $1.99 \mathrm{e} 11 \mathrm{~Pa}$. Poisson's ratio is 0.3 , yield limit is $205 \mathrm{MPa}$, and tangent modulus is $7000 \mathrm{MPa}$.

Free grid was used to mesh. The press should be load as time-related pressure on every element of the finite element model. So we need to create a component(named E1) that include all elements and setup a two-dimensional array include time and pressure. Fig. 3 shows the finite element model after the pressure array was loaded on E1 [8]. Setup the solution time and some other necessary parameter based on the loading process. Then the strain contours could be got through simulation in post processor [9]. The effect is given in Fig. 4.

To certify the reliability of finite element analysis, a test panel with the same parameters was used to conduct an experiment and compare the result with analysis data. Weld the structure of the dimple jacket in accordance with the requirements of manufacturing and distend the jacket by increasing the bulging pressure gradually. The bulging process should be stopped immediately if the pressure exceeded the extremity that would lead up to the deformation of the substrate. According to the machining 
requirement of dimple jacket, the bulging speed could not exceed $0.1 \mathrm{MPa} / \mathrm{min}$. Bulge the jacket and checked the jacket state when the pressure reached 1.4 MPa. Keep the pressure value for long enough time. Then measure the bulging height once when the pressure increased every 0.2 $\mathrm{MPa}$. Stop pressing when the pressure reaches $2.0 \mathrm{MPa}$ and make a full review after keeping long enough time [10]. The effect of practical bulging is given in Fig. 5. Then we can unload the pressure after keeping it at 2.0 $\mathrm{MPa}$ for long enough time. In the end we just need to measure the final bulging height of the jacket.

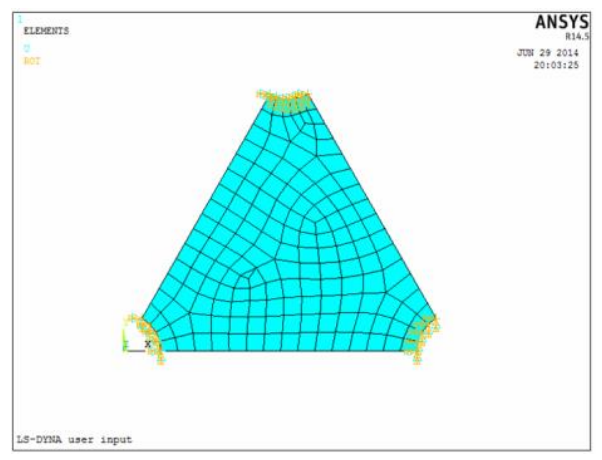

Fig. 2. The definition of constraints

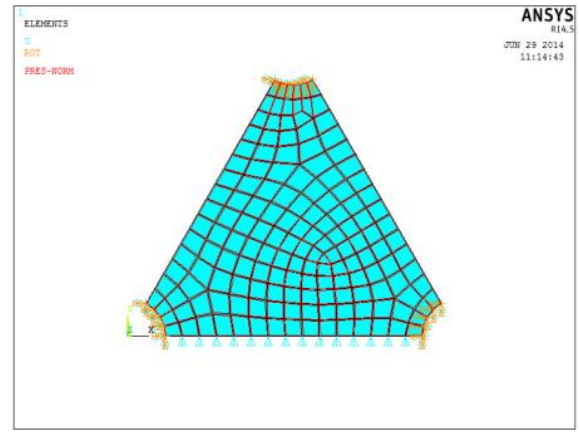

Fig. 3. Loading effect of finite element model

\section{RESULTS}

As we can see in Fig. 5, the result of finite element analysis shows that the maximum value of final bulging height is $6.2499 \mathrm{~mm}$, and the highest point is located in the center of the triangle. That means the farther away from welding circle, the higher of the bulging height. According to the experiment of test panel, we can measure the jacket to get the bulging height. The results show that the practical height is $6.12 \mathrm{~mm}$. The error between analysis value and practical value is $2.12 \%$. What need to be explained is both the analysis height and practical height are transient values under certain bulging pressure, and part of the elastic height may recover after unloading.

\section{DISCUSSION}

We have made a successful example of a model with certain parameters. While in order to obtain the relation between bulging height and bulging pressure and to verify the finite element analysis of bulging value (including bulging height and bulging pressure) accord with the practical bulging value, we have to conduct the other necessary analysis and experiment in different parameter models. Table 2 provides model parameter combinations that are most commonly used in process industry, we need to do the same operation for these models.

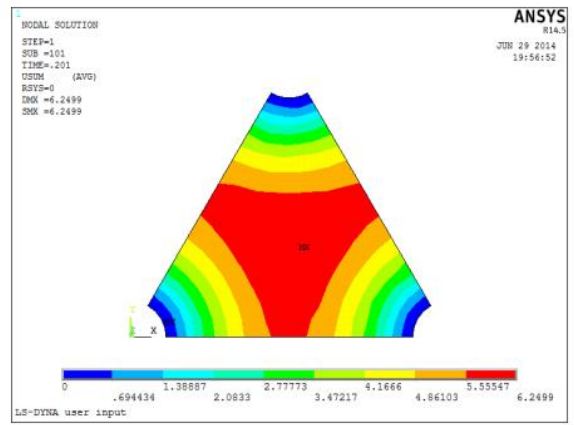

Fig. 4. Strain contours of the jacket

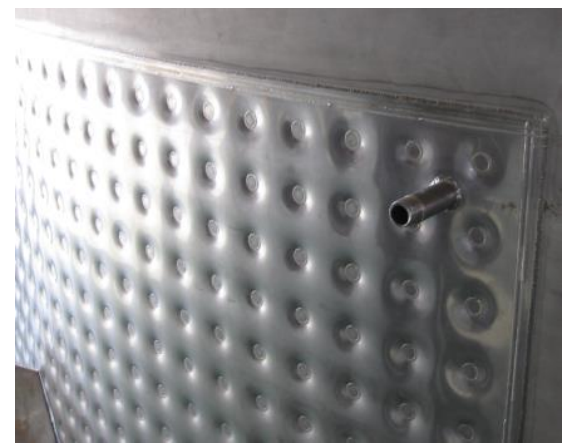

Fig. 5. Effect of practical bulging

The results of analysis and experiment are shown in Table 3. We can see that several models with different parameters were analysed. The edge length ranges from $60 \mathrm{~mm}$ to $100 \mathrm{~mm}$, the diameter of the welding circle from $15 \mathrm{~mm}$ to $20 \mathrm{~mm}$, the thickness of the jacket from $1.0 \mathrm{~mm}$ to $1.2 \mathrm{~mm}$. So we can obtain the rule of the bulging pressure changing with different parameter. We can also know whether the analysis value is accurate through comparing it with experiment value.

Table 3. Relations between bulging height and pressure

\begin{tabular}{|c|c|c|c|c|}
$\begin{array}{c}\text { Heat } \\
\text { transfer } \\
\text { medium }\end{array}$ & $\begin{array}{c}\text { Model } \\
\text { parameters }\end{array}$ & $\begin{array}{c}\text { bulging } \\
\text { pressure, } \\
\mathrm{MPa}\end{array}$ & $\begin{array}{c}\text { bulging } \\
\text { height, } \\
\mathrm{mm}\end{array}$ & $\begin{array}{c}\text { Practical } \\
\text { height, } \\
/ \mathrm{mm}\end{array}$ \\
\hline Alcohol & $88 \_20 \_1.0$ & 2.0 & 6.25 & 6.12 \\
& 100_20_1.0 & 1.8 & 5.84 & 5.66 \\
\hline Water & 88_20_1.2 & 2.4 & 5.03 & 4.92 \\
vapor & 100_20_1.2 & 2.0 & 5.52 & 5.34 \\
\hline Liquid & 60_15_1.2 & 4.2 & 5.0 & 4.89 \\
ammonia & 70_15_1.2 & 3.8 & 5.1 & 5.02 \\
\hline
\end{tabular}

\section{CONCLUSION}

This paper takes laser welded dimple jacket as the object of study and analyses different parameter models to obtain the relation between bulging height and pressure of the jacket honeycomb. Through the study and experiment we can get the following conclusions.

1. Strain contours of finite element model were achieved through calculation, which indicated that the maximum displacement point located in the centre of the triangle model. We can also get the height value by the analysis result.

2. The bulging height increases with the increasing of the bulging pressure and the space of honeycomb. And it will decrease when the thickness of jacket plate is 
changing larger. Table 3 can be used as the preferred series to instruct the machining process.

3. The results of experiment on different parameter test plates show that the errors between analysis value and practical value are all under $10 \%$. According to the accuracy requirements of the jacket manufacture and the error estimates of finite element analysis, the result obtained through ANSYS/LS-DYNA is able to meet the accuracy requirements of engineering calculation [11].

4. The using of finite element analysis in studying relation between bulging height and pressure can enhance the productivity and reduce expenses. Contrasting with repeating prototype testing, this approach is better in advancement and efficiency. It provides a new idea to research the laser welded dimple jacket.

\section{Acknowledgments}

The research is supported by Program for Changjiang Scholars and Innovative Research Team in University (IRT1266), Shandong Province Natural Science Foundation, China (No. ZR2011EEM014) and Program for Scientific research innovation team in Colleges and universities of Shandong Province.

\section{REFERENCES}

1. Xueli, H., Feng, P. Welding of Dimple Jacket Vessel Pressure Vessel Technology 18 (2) 2001: pp. 53-55.

2. Yanni, C. Structure Design and Comparison of jacket Vessel Nonferrous Metallurgical 6 2013: pp. 17-20.
3. Cong, H., Shijian, Y. Hydroforming of an Aluminum Alloy Spherical Shell Pressure Vessel Technology $22(8)$ 2005: pp.10-13.

4. Fei, W., Hongbin, L., Zhitao, J., Lingbing, F. Forming Process Simulation of Half-Honeycomb Profile Strip Based on LS- DYNA Science \& Technology Review 16 (13) 2008: pp. $49-51$.

5. Bin, L., Qinghai, J. A Study on the Experimentation and Analysis of Function Force Concerning Curve Thin Plate tf The Curtain Wall with Hydraulics Form Machine Tool \& Hydraulics 5 2003: pp. 96-98.

6. Haiying, D., Xia, Y. The Three-Dimensional Stress Analysis and Design of Dimple Jacket Structure Based on ANSYS Cereals and Oils Processing 4 2009: pp. $148-151$.

7. Changliang, S. 3D Dynamic Simulation Analysis of ThinWall Parts Forming Process Based on ANSYS/LS-DYNA. Northeastern University, Shenyang, 2008.

8. Yuanquan, Ch., Jian, G. Analysis of Deformation on The Blade During The Machining Process Based on ANSYS/LSDYNA Machinery Design \& Manufacture 10 2010: pp. $101-103$.

9. Weibo, Z. Research on the Strength Analysis of Laser Welding Dimple Jacket. Zhejiang University, Hangzhou, 2008.

10. Baoqing, L., Peng, L., Xinghua, L., Weibo, Z. Experiment And Finite Element Analysis on Pressure Distention of Laser Welding Dimple Jacket Journal of Zhejiang University (Engineering Science) $45(3)$ 2008: pp. $571-575$.

11. Xiaoyu, W., Qingyun, W., Hongbo, Y., Huiyang, H. Discussion on Determination Method of Finite Element Analysis Result Machine Design and Research 19 (6) 2003: pp. $13-15$. 